\title{
Angela Bernardo, Alessandro Saggioro (eds), I Principi di Toledo e le religioni a scuola
}

Traduzione, presentazione e discussione dei Toledo Guiding Principles on Teaching about Religions and Beliefs in Public Schools (OCES/ODIHR), Aracne, Roma 2015, ISBN 978-88-548-8897-5, pp. 284, € 17

Chiara Di Serio

\section{(2) OpenEdition}

Edizione digitale

URL: http://journals.openedition.org/mythos/535

DOI: $10.4000 /$ mythos. 535

ISSN: 2037-7746

Editore

Salvatore Sciascia Editore

\section{Edizione cartacea}

Data di pubblicazione: 1 dicembre 2016

Paginazione: 238-241

ISBN: 978-88-8241-476-4

ISSN: $1972-2516$

Notizia bibliografica digitale

Chiara Di Serio, « Angela Bernardo, Alessandro Saggioro (eds), I Principi di Toledo e le religioni a scuola », Mythos [Online], 10 | 2016, online dal 24 septembre 2019, consultato il 25 septembre 2020. URL

http://journals.openedition.org/mythos/535; DOI : https://doi.org/10.4000/mythos.535 


\section{Angela Bernardo e Alessandro Saggioro (eds)}

I Principi di Toledo e le religioni a scuola. Traduzione, presentazione e discussione dei Toledo Guiding Principles on Teaching about Religions and Beliefs in Public Schools (OCES/ODIHR)

Aracne, Roma 2015, ISBN 978-88-548-8897-5, pp. 284, €17

Chiara Di Serio - Università di Roma La Sapienza - chiara.diserio@virgilio.it

II libro pubblicato nel dicembre del 2015 a cura di Angela Bernardo e Alessandro Saggioro costituisce la presentazione e la traduzione in italiano di un documento pubblicato nel 2007 dall'ODIHR dell'OSCE (Organization for Security and Cooperation in Europe). L'edizione è nata come significativa testimonianza di un vivace dibattito presente in ambito accademico all'interno degli interessi più recenti degli studi storico-religiosi in Italia. II problema dell'insegnamento delle religioni, laicamente inteso, scientificamente determinato e pluralisticamente orientato, non può più essere messo da parte ed è ormai questione sempre più centrale nella nostra società post-moderna, liquida e culturalmente globalizzata. L'edizione italiana dei Principi guida di Toledo esprime una concreta volontà di affrontare il nodo spinoso (specialmente in Italia) della disciplina riguardante lo studio delle religioni nelle scuole. Dei due curatori, Alessandro Saggioro si è da tempo occupato della questione ${ }^{1}$ e intende naturalmente rilanciare il dibattito sul tema.

La struttura dell'opera contiene un'Introduzione, di cui conviene ripercorrere la prima parte per comprendere il senso dell'intero lavoro. In essa si spiega come il documento dell'OSCE pubblicato per la prima volta nel 2007 non abbia avuto in Italia alcun riscontro. Esso voleva essere soprattutto un atto politico: nato in un ambito in-

1 Si vedano gli studi di A. Saggioro, in particolare Più problemi che soluzioni. Documenti per una didattica storico-religiosa, EuRoma, Roma 1998 e M. Giorda e A. Saggioro, La materia invisibile. Storia delle religioni a scuola. Una proposta, EMI, Bologna 2011. ternazionale, avrebbe dovuto trovare ascolto nei contesti locali per iniziare una discussione su un tema di assoluto interesse per la convivenza, l'integrazione, l'interazione tra culture diverse. Tuttavia gli obiettivi prevalenti della politica si concentrano spesso sulle dinamiche economiche e quindi poco spazio viene dato, sul piano dell'attuazione di una vera inclusione, ai fondamenti da costruire che sono eminentemente umani e attinenti alla sfera dei valori. I Principi di Toledo hanno avuto lo scopo di individuare linee di convergenza su una tematica "sensibile", poiché anche nell'orizzonte della formazione si affacciano costantemente complesse forme di pluralismo.

Dal momento che è necessaria una continua ridefinizione, a livello delle istituzioni internazionali, ma anche nazionali e locali, dei criteri di integrazione, la parola d'ordine può essere una sola: mediazione. Nelle scelte dei governi, europei e d'oltremare, si alternano operosità e momenti di inazione. Ma, nel frattempo, prolificano tensioni, estremismi, conflitti e le "buone pratiche" non fanno storia. II XXI secolo ha avuto un'apertura drammatica con i fatti dell'11 settembre 2001 e viviamo conflitti che ormai si possono definire globali. A dispetto di ciò, esistono delle dinamiche di relazioni internazionali che vogliono riproporre principi di pace e convivenza, che diano una risposta concreta all'esigenza primaria di interazione tra culture diverse.

I Principi di Toledo fanno parte delle "trincee della ragionevolezza" contro l'affermarsi delle politiche di guerra. Essi hanno un valore assoluto in senso pacifista. La loro funzione media l'operatività dei sistemi educativi che devono di necessità raffrontarsi 
con dinamiche culturali diverse. La storia non può non porsi come strumento trasversale di superamento dei confini ideologici e nazionali, dice A. Saggioro (p. 26), e la conclusione è che la soluzione allo stato attuale di crisi mondiale può essere trovata solo in un diffuso movimento di confronto e di ricostruzione culturale.

Sempre nell'Introduzione, A. Bernardo sottolinea come i Principi di Toledo facciano parte di una strategia che vuole considerare l'elemento religioso causa di promozione della coesione sociale e come nascano nel quadro di una generale difesa dei diritti umani e delle libertà fondamentali dell'uomo, e rileva che essi hanno preparato la strada ad un nuovo interesse delle nazioni europee verso il processo di unificazione ormai da tempo caldeggiato da più parti. II testo dei Principi, infatti, ha come suo primo obiettivo quello di ri-mediare situazioni di conflitto. II ruolo della formazione viene in seguito considerato fondamentale per la prevenzione in fatto di ostilità nei confronti dell'altro, del diverso, dello straniero. L'Autrice poi delinea in sintesi il piano dell'opera, illustrando il contenuto dei singoli capitoli del testo tradotto.

La prima parte del volume, che prepara la lettura del documento, contiene tre saggi redatti da autorevoli studiosi italiani, esperti del settore di insegnamento della o sulla religione. II primo, particolarmente interessante, è di Brunetto Salvarani, docente di Teologia della Missione e del Dialogo presso la Facoltà Teologica dell'Emilia-Romagna. In esso viene tratteggiato lo scenario contemporaneo della società attuale. In primis si illustra come ormai il Dio dell'Europa sia un Dio plurale. Le religioni sono un work in progress ed è possibile per qualsiasi individuo scegliere di essere ateo, di seguire un'ortodossia, di cambiare professione, o di ritagliarsi un proprio personale percorso, seguendo un processo di soggettivizzazione delle credenze sempre più presente e verificabile. Vi è una pluralità di scelte possibili che da una parte rassicura la libertà degli individui, ma dall'altra dà meno certezze ai singoli. Le grandi istituzioni delle religioni tradizionali, in particolare quella cristiana, vedono vacillare il loro secolare costrutto, a confronto con una mobilità spaventosa del mondo contemporaneo, in cui prevalgono il cambiamento, la flessibilità, le spinte centrifughe, la complessità. Secondo Salvarani il cristianesimo si sta trasformando, ma non è detto che stia scomparendo, e anche i musulmani dovranno ripensare ai loro valori tradizionali. Si assisterà al sorgere di nuove forme di fede e di pratiche religiose. La società in cui viviamo - riferisce Salvarani mette in crisi il modello educativo dell'uno, cioè del legame con un solo territorio, una sola lingua, una sola religione, una sola cultura. Ma negli ultimi trent'anni tale modello, tipico dello stato nazionale, è stato reso problematico da diversi fattori, che vengono sinteticamente elencati e spiegati da Salvarani2: secolarizzazione; differenziazione e pluralizzazione; complessificazione; individualizzazione; variabilità ed accelerazione; astrazione e dematerializzazione; eccedenza simbolica; mediatizzazione (pp. 42-43).

In questa dimensione così frammentaria e frantumata, il disorientamento sembra essere la condizione più diffusa. Per rispondere a questa situazione lo Stato sembrerebbe essere tentato dalla logica della paura: lascia che siano gli individui a trovare soluzioni personali a una crisi che invece è ormai sistemica. II tutto viene alimentato dalla strategia della tensione messa in atto da attività criminali, dal comportamento antisociale delle classi più basse, dal terrorismo globale. Secondo Salvarani la costruzione di un mondo più equo, sostenibile, rispettoso delle differenze è un compito primario della politica, ma è anche una irrinunciabile sfida educativa (p. 45). II modo dell'educazione deve prendere in considerazione almeno: il proprio ruolo; la propria missione; il senso dell'interazione; le modalità di interazione tra generazioni; le modalità di costruzione

2 Naturalmente la fonte principale di Salvarani è qui il pensiero di Z. Bauman, di cui si vedano almeno Modernità liquida, Roma-Bari 2002 e Il disagio della postmodernità, Milano 2002. 
di una cultura plurale. Occorrerebbe una rivoluzione del pensiero, del metodo e delle forme di relazione. Bisognerebbe arrivare a formulare una nuova idea di cittadinanza glocale, con un ambito di relazione tra globale e locale, uno scenario in cui le differenze si incontrano, si scontrano e si ibridano, un luogo di scomposizione e di ricomposizione.

La questione della laicità contemporanea rappresenta il punto focale di una via di ritorno alla "normalità". II fatto è che nella società post-secolare Dio è tornato, come dice Salvarani. II ritorno al sacro è stato definito post-secolarismo e risponde alla crescente solitudine e disorientamento dell'individuo. In Italia permane ancora una forma di conflitto ideologico tra i fedeli alla Chiesa e i combattenti per la laicità, ma questa è una battaglia inutile e dannosa. L'unica via percorribile è quella dell'apertura da entrambe le parti. Si potrebbe arrivare a una nuova cultura laica, che lasci a margine ogni integralismo, ma che accetti le religioni come complementari allo Stato liberale ( $p$. 48). La religione ha ormai un rilievo pubblico di cui lo Stato liberale dovrebbe far tesoro; essa è una forza in continua crescita, che segna l'avvento di un'epoca post-secolare. Infine - conclude Salvarani - il piano di riforma della scuola italiana sembra essere improntato ad una soluzione fin troppo semplice a fronte di un problema assai complesso. La strada mitica della semplificazione della complessità non esiste. II compito primario della scuola allora è quello di dare risposte competenti a un'istanza di multiculturalità e crescita nel pluralismo. Non sono più proponibili modelli culturali identitari, ma ciascuno di noi è chiamato ad essere straniero a se stesso (p. 51) per aprirsi al dialogo, all'incontro, alla mediazione e costruire un nuovo linguaggio condiviso.

Seguono altri due lavori, rispettivamente di Flavio Pajer e Mariachiara Giorda. II primo, professore di Pedagogia e Didattica delle Religioni presso la Pontificia Università Salesiana di Roma, si è occupato di fornire un'analisi accurata dei criteri orientatori che hanno ispirato i Principi guida. Egli precisa che si parla nel documento di insegnamento about religions and beliefs, laddove l'oggetto religioso è declinato al plurale, e il termine beliefs implica un ambito che coinvolge anche le fedi secolari. Viene sottolineato che essi insistono sulla necessità di una conoscenza oggettiva, critica, comparata dei fenomeni religiosi e delle loro fonti, della loro genesi storica, delle loro ricadute in ogni campo. Premono sulle condizioni culturali e formative da promuovere perché gli studenti imparino a vivere nella diversità, ma anche a condividere una tavola di valori comuni che siano alla base della convivenza. Lo stesso studioso poi elenca le Conclusioni e le Raccomandazioni presenti alla fine del testo, per spiegare che quelle sono le risposte dirette alla domanda: a cosa serve l'insegnamento di una disciplina che riguardi lo studio delle religioni? Nell'ultima parte del suo studio, Pajer auspica un nuovo percorso curricolare in Italia sulla cultura religiosa che possa: garantire un apprendimento interreligioso; comprendere più a fondo le differenze religiose; analizzare le nuove forme di religiosità e spiritualità; portare a costruire ed esprimere la propria identità come ad accettare quella degli altri. Infine, viene indicato in maniera specifica come dovrebbe configurarsi l'istruzione in fatto di religione in sette punti (e in questo caso viene da osservare che la proposta di Pajer appare del tutto innovativa). Essa deve diventare un insegnamento: 1) relativo alle religioni e agli orizzonti non religiosi; 2) religioso, di carattere prevalentemente oggettivo; 3) pluralista, aperto alle diversità; 4) destinato alla totalità degli studenti e quindi a carattere curricolare; 5) avente come obiettivi quelli della educazione interculturale; 6 ) inserito in un ambiente di saperi disciplinari aggiornati riguardo alle più attuali ricerche religiose; 7 ) esercitato da un docente curricolare, formato in istituzioni accademiche pubbliche.

Nell'ultimo dei tre saggi che integrano il testo dei Principi M. Giorda, docente di Storia delle Religioni presso l'Università di Bologna, traccia un quadro multiprospettico in cui l'ambito dell'educazione alla cittadinan- 
za come disciplina specifica già presente nell'ordinamento scolastico vigente, - i cui obiettivi primari sono quelli di diffondere tra i giovani la cultura della democrazia, di contribuire alla lotta contro la violenza e di promuovere la coesione sociale ed il bene comune - potrebbe costituire la base di un percorso didattico in cui trovi ragion d'essere la Storia delle Religioni. A corredo della proposta, M. Giorda inserisce nel suo lavoro anche un'ipotesi didattica di insegnamento della disciplina, enucleando le priorità specifiche: l'approfondimento della terminologia storico-religiosa; la lettura e l'interpretazione dei testi sacri, dei segni, dei simboli e delle immagini religiose; la conoscenza delle maggiori aree di individuazione dei fenomeni storico-religiosi, distinguendo tra tutte le diverse definizioni di religioni (primitive, fondate, universali, etniche, sètte, ecc.); la conoscenza delle linee storiche di sviluppo delle tradizioni religiose che hanno maggiore interesse per il mondo occidentale; la capacità di cogliere connessioni tra tradizioni religiose diverse; la capacità di giudizio sulle esperienze religiose e l'attivazione di una coscienza critica. La studiosa prosegue enumerando in maniera dettagliata le competenze da trasmettere agli studenti nel quadro dell'insegnamento sulle religio$\mathrm{ni}$, in riferimento ai vari campi del sapere e della formazione in genere, e infine esplicita anche i singoli contenuti da proporre per i cinque anni delle scuole superiori in Italia. II suggerimento di M. Giorda è sicuramente un valido percorso operativo.

Nella seconda parte del volume, troviamo il testo del documento, che già nella sua versione originale, presentava una Sintesi, assai utile per comprendere la portata dei contenuti. I Principi di Toledo nei loro sei capitoli fissano la necessità di un insegnamento relativo alle religioni e alle creden$z e$, il quale possa rientrare in un quadro più ampio di attuazione dei diritti umani. La libertà di religione appare ormai fattore imprescindibile per la realizzazione di un pieno riconoscimento del valore della persona. Ma dal momento che la nostra società post-moderna vede l'avanzare e l'affermarsi di un panorama di religioni e credenze alquanto complesso e diversificato, l'unica via possibile al perseguimento di tale libertà è il confronto, l'interscambio, la comparazione, comprendenti sia la differenziazione che l'assimilazione (o l'uniformazione).

Raggiungere un livello di coscienza ugualmente vivo in tutti gli individui, che pratichino credenze diverse, anche in un orizzonte secolarizzato, o religioni differenti, sulla pari dignità di ogni pensiero religioso, includendo qualunque spessore valoriale o fondativo, non è facile e necessita di un percorso formativo ben determinato e privo di incertezze. Per questo sarebbe doveroso oggi pensare alla formazione di docenti preparati ad affrontare l'insegnamento di una disciplina come la storia delle religioni (declinata al plurale) e non più dell'unica religione possibile. La preparazione degli insegnanti dovrebbe essere laica, pluralista, multiprospettica, multiculturale e capace di dare valore nella giusta misura a idee e istanze anche divergenti. Parallelamente i curricula proposti per gli studenti dovrebbero prevedere al loro interno contenuti e metodi che tengano conto delle idee religiose e non religiose in maniera inclusiva e non discriminatoria. I piani di studio dovrebbero fare particolare attenzione alle trasformazioni della società contemporanea rispecchiando questioni globali ma anche locali, collegando di volta in volta il pluralismo religioso e laico alle manifestazioni locali delle singole realtà incontrate.

Questi sono in nuce gli elementi che stanno alla base dei Principi guida fondamentali presenti in apertura del documento. Da parte nostra, considerata la serietà degli intenti dei Principi di Toledo e condividendo l'urgenza di un approccio diverso e nuovo all'insegnamento delle religioni, non possiamo far altro che auspicare la realizzazione di un vasto e ambizioso progetto come questo, nella speranza che l'orizzonte dell'uno si apra alla via del molteplice. 